\title{
Therapeutic Effect of Three-Dimensional Conformal Radiotherapy Combined with Tegafur Gimeracil and Oteracil Potassium in the Treatment of Locally Advanced Esophageal Cancer
}

\author{
Xiaoyan Yan, Hongsheng Yu* \\ Qingdao University, Qingdao, China \\ Email:^825183843@qq.com
}

How to cite this paper: Yan, X.Y. and Yu, H.S. (2019) Therapeutic Effect of ThreeDimensional Conformal Radiotherapy Combined with Tegafur Gimeracil and Oteracil Potassium in the Treatment of Locally Advanced Esophageal Cancer. Open Access Library Journal, 6: e5303. https://doi.org/10.4236/oalib.1105303

Received: February 28, 2018

Accepted: March 19, 2019

Published: March 22, 2019

Copyright $\odot 2019$ by author(s) and Open Access Library Inc.

This work is licensed under the Creative Commons Attribution International License (CC BY 4.0).

http://creativecommons.org/licenses/by/4.0/

\begin{abstract}
Objective: To observe the therapeutic effect of three-dimensional conformal radiotherapy combined with Tegafur Gimeracil and Oteracil Potassium in the treatment of locally advanced esophageal cancer. Method: The patients with locally advanced esophageal cancer admitted to our hospital were randomly divided into observation group and control group. The control group was treated with three-dimensional conformal radiotherapy alone; the observation group, three-dimensional conformal radiotherapy combined with tegafur was used. Result: In the observation group, the patient's disease control rate was $89.74 \%$, and in the control group, the patient's disease control rate was $71.79 \%$. The results of the two groups were compared and the difference was statistically significant $(\mathrm{p}<0.05)$. In the observation group, the patient's leukopenia rate was $38.46 \%$, the thrombocytopenia rate was $35.90 \%$, the incidence of nausea and vomiting was $53.85 \%$, and the quality of life was $(85.20 \pm$ $1.59)$ points. There was no significant difference ( $p>0.05)$ compared with the control group. The local recurrence rate of the observation group was $10.26 \%$, and the difference was significant compared with the control group $(\mathrm{p}<$ 0.05). Conclusion: The treatment of locally advanced esophageal cancer by three-dimensional conformal radiotherapy combined with Tegafur Gimeracil and Oteracil Potassium treatment can effectively improve the control rate of the disease, and its clinical application value is very significant.
\end{abstract}

\section{Subject Areas}

Oncology 


\section{Keywords}

Three-Dimensional Conformal Radiotherapy, Tegafur, Gimeracil and Oteracil Potassium, Local Advanced Esophageal Cancer

\section{Introduction}

Esophageal cancer is a malignant tumor of the digestive system that is very common in the clinic. The main patients are mostly elderly. The conventional treatment of esophageal cancer is mainly through the combination of concurrent radiotherapy and systemic intravenous chemotherapy. Although the effect of this method is obvious, in the course of treatment, patients will suffer greater pain, and there will be more complications that will occur. Studies have shown that the effectiveness of using three-dimensional conformal radiotherapy combined with Tegafur Gimeracil and Oteracil Potassium for the treatment of locally advanced esophageal cancer is relatively higher. In this study, during the period from December 2016 to December 2017, 78 patients with locally advanced esophageal cancer admitted to our hospital were randomly selected as samples to observe the clinical efficacy of different treatments.

\section{Materials and Methods}

\subsection{General Materials}

The patients with locally advanced esophageal cancer admitted to our hospital from December 2016 to December 2017 were randomly divided into observation group and control group, and each group has 39 cases. In the observation group, patient data include: gender: male/female $=19 / 20$, age $(38-91)$ years old, mean $(59.76 \pm 1.39)$ years old. In the control group, patient data include: gender: male/female $=20 / 19$, age $(40-80)$ years, mean $(59.85 \pm 1.42)$ years old. This study has been reviewed and approved by the affiliated hospital of Qingdao University of the Economic and Technological Development Zone. There were no significant differences between the two groups and they were comparable $(\mathrm{p}>0.05)$.

\subsection{Method}

\subsubsection{Three-Dimensional Conformal Radiotherapy}

In the control group, patients were treated with three-dimensional conformal radiotherapy alone: 1) fixed position, continuous CT scan of mediastinum, layer thickness $4 \mathrm{~mm}$. 2) gross tumor volume (GTV) and determine the clinical target volume (CTV), planned target volume (PTV), and organ at risk (OAR). 3) Design conformal field, radiotherapy with $95 \%$ dose curve. 4) The dose of radiotherapy is $2 \mathrm{~Gy}$, and the total dose is $60-66 \mathrm{~Gy}$, for a total of $30-33$ times. The lung volume should be $\leq 25 \%$ of the lung volume and the spine dose should be $<60$ Gy. 


\subsubsection{Medicinal Methods of Tegafur Gimeracil and Oteracil Potassium}

In the observation group, patients were treated with three-dimensional conformal radiotherapy combined with Tegafur Gimeracil and Oteracil Potassium: 1) Three-dimensional conformal radiotherapy: The method was consistent with the control group. 2) Usage and dosage of Tegafur Gimeracil and Oteracil Potassium: Take the drug on the first day of radiotherapy, the dose is $40 \mathrm{mg} / \mathrm{m}^{2}$, bid, and take it orally after breakfast and dinner. After 28 days of continuous medication, rest for 14 days, and then repeated treatment with the above dose. After 4 cycles of treatment, observe the therapeutic effect.

\subsection{Observation Index}

The disease control rate of the two groups was observed, including four indicators which were CR (complete response), PR (partial response), SD (stable disease), and PD (progressive disease). The incidence of adverse reactions and quality of life were observed in the two groups. The quality of life was evaluated by using the SF-36 scale [1]. If the score is higher, it means the quality of life is greater.

\subsection{Statistical Analysis}

The experimental data were calculated using SPSS Version 19.0 software. According to the analysis, the count data is expressed as a percentage, and the comparison between groups is $\chi^{2}$-test. Results were defined as statistically significant at $\mathrm{P}<0.05$.

\section{Results}

\subsection{The Comparison of Clinical Efficacy between Two Groups}

The control rate of the treatment group was significantly higher than that of the control group, and the difference was statistically significant $(\mathrm{P}<0.05)$, as shown in Table 1.

\subsection{The Comparison of the Incidence of Adverse Reactions and Quality of Life between the Two Groups}

The incidence of adverse reactions and quality of life of was no difference between two groups, but the local recurrence of the treatment group was significantly higher than that of the control group, and the difference was statistically significant $(\mathrm{P}<0.05)$, as shown in Table 2.

\section{Discussion}

Because the mortality rate of patients with locally advanced esophageal cancer is

Table 1. Disease control rates of the two groups of patients.

\begin{tabular}{cccccc}
\hline Group type & CR (n/\%) & PR (n/\%) & SD (n/\%) & PD (n/\%) & Control rate (n/\%) \\
\hline Observation group & $15(38.46)$ & $12(30.77)$ & $8(20.51)$ & $4(10.26)$ & $35(89.74)$ \\
Control group & $2(5.13)$ & $15(38.46)$ & $11(28.21)$ & $11(28.21)$ & $28(71.79)$ \\
p & & \multicolumn{5}{c}{$<0.05$} \\
\hline
\end{tabular}


Table 2. The incidence of adverse reactions and quality of life in the two groups of patients.

\begin{tabular}{|c|c|c|c|c|c|}
\hline \multirow{2}{*}{$\begin{array}{c}\text { Group type } \\
-\end{array}$} & \multicolumn{3}{|c|}{ Adverse reactions } & \multirow{2}{*}{$\begin{array}{l}\text { Quality of life } \\
\text { (Points) }\end{array}$} & \multirow{2}{*}{$\begin{array}{l}\text { Local recurrence } \\
\qquad(\mathrm{n} / \%)\end{array}$} \\
\hline & Decrease of leukocyte & Decrease of platelet & Feel sick or vomit & & \\
\hline Observation group & $15(38.46)$ & $14(35.90)$ & $21(53.85)$ & $85.20 \pm 1.59$ & $4(10.26)$ \\
\hline Control group & $14(35.90)$ & $14(35.90)$ & $20(51.28)$ & $84.19 \pm 0.62$ & $11(28.21)$ \\
\hline $\mathrm{p}$ & $>0.05$ & $>0.05$ & $>0.05$ & $>0.05$ & $<0.05$ \\
\hline
\end{tabular}

usually high, treatment with a radiotherapy method can effectively inhibit the progression of the disease and reduce the risk of recurrence and metastasis [2]. This has a positive effect on prolonging the lifespan of patients. Three-dimensional conformal radiotherapy is a method of radiotherapy that allows for the setting of conformal fields prior to treatment. On this basis, high dose areas can be distributed in the lesion site. This method can further reduce the dose of radiation outside the tumor and achieve the purpose of effectively killing tumor cells while protecting healthy organs and tissues around the tumor [3].

Tegafur Gimeracil and Oteracil Potassium is a chemotherapeutic drug and is a new combination of 5-FU (5-fluorouracil). The drug consists of tegafur, gimeracil, and oteracil potassium. The ratio of the three drugs is 1:0.4:1. The mode of administration of Tegafur Gimeracil and Oteracil Potassium is mainly oral [4]. When the drug enters the human body, it can be rapidly converted to 5-FU to achieve the purpose of chemotherapy. On the basis of three-dimensional conformal radiotherapy, patients were given chemotherapy with Tegafur Gimeracil and Oteracil Potassium, in order to effectively inhibit the accelerated proliferation of cells. Thereby, the tumor volume can be shortened, and the damaged part of the esophagus can be repaired. Through the research in this paper, we can find that patients with locally advanced esophageal cancer are treated with Tegafur Gimeracil and Oteracil Potassium combined with three-dimensional conformal radiotherapy, their disease control rate is $89.74 \%$. Compared with the treatment with three-dimensional conformal radiotherapy alone, the difference was statistically significant $(\mathrm{p}<0.05)$. This result indicates that if the two methods are combined, the disease control rate of patients with locally advanced esophageal cancer can be effectively improved. Through the observation of adverse reactions in patients, the reduction rate of leukocytes in this group of patients was $38.46 \%$, the reduction rate of platelet was $35.90 \%$, the incidence of nausea and vomiting was $53.85 \%$, and the quality of life was $(85.20 \pm 1.59)$. There was no significant difference between this result and the treatment with three-dimensional conformal radiotherapy alone $(\mathrm{p}>0.05)$. This result suggests that the use of Tegafur Gimeracil and Oteracil Potassium in the treatment does not increase the risk of adverse reactions. By observing the patient's recurrence, we found that the patient's local recurrence rate was $10.26 \%$ by treating patients with three-dimensional conformal radiotherapy combined with Tegafur Gimeracil and Oteracil Potassium. This result was compared with the three-dimensional conformal radiotherapy alone and was significantly different $(\mathrm{p}<0.05)$. The above 
findings suggest that three-dimensional conformal radiotherapy combined with Tegafur Gimeracil and Oteracil Potassium in the treatment of locally advanced esophageal cancer can help reduce the local recurrence rate, which is of great significance for prolonging the life of the patient.

\section{Conclusion}

In conclusion, three-dimensional conformal radiotherapy combined with Tegafur Gimeracil and Oteracil Potassium can effectively improve the disease control rate and reduce the local recurrence rate. Its clinical application value is significant.

\section{Conflicts of Interest}

The authors declare no conflicts of interest regarding the publication of this paper.

\section{References}

[1] Wang, Z., Dong, H., Wang, Q., Zhang, L., Wu, X., Zhou, Z., Yang, L. and Huang, D. (2019) Effects of Electroacupuncture on Anxiety and Depression in Unmarried Patients with Polycystic Ovarian Syndrome: Secondary Analysis of a Pilot Randomised Controlled Trial. Acupuncture in Medicine.

[2] He, F.J. and Zhou, F. (2018) Observation of Clinical Efficacy of Magnetotherapy Combined with Radiotherapy in the Treatment of Cervical or Supraclavicular Metastatic Lymph Nodes of Lung Cancer or Esophageal Cancer. Medical Theory and Practice, 31, 2110-2112.

[3] Wu, S. (2018) Clinical Observation of Three-Dimensional Conformal Radiotherapy Combined with Thalidomide in the Treatment of Esophageal Cancer and Its Effect on Serum MIP-3, VEGF and VEGFR-1. Journal of Practical Cancer, 33, 1065-1068.

[4] Xu, Y.F. (2018) Dosimetric Comparison of Three-Dimensional Conformal Radiotherapy and Intensity-Modulated Radiotherapy in Radical Radiotherapy for Middle Thoracic Esophageal Cancer. Medical Information, 31, 73-76. 